\title{
The importance of severe cytolysis syndrome in hypertrophic cardiomyopathy in children
}

\author{
Flavia-Cristina Al-Akel' ${ }^{1}$, Amalia Fagarasan ${ }^{2,3}$ \\ ${ }^{1}$ Paediatric Clinic 1, Emergency County Hospital, Targu Mures, Romania \\ ${ }^{2}$ University of Medicine, Targu Mures, Romania \\ ${ }^{3}$ Department of Paediatric Cardiology, Cardiovascular and Transplant Emergency Institut of \\ Targu Mures, Romania
}

\begin{abstract}
Hypertrophic cardiomyopathy is the second most frequent cardiomyopathy in children and it counts as a genetic disorder for a high number of cases, with widely variable clinical presentation. The disease can be recognized in all age groups and has an unpredictable prognosis, especially if undiagnosed on time.

We present the case of a two and a half years old male toddler diagnosed at territorial level with systolic heart murmur, who manifested mild fatigue, whose echocardiographic examination showed obstructive hypertrophic cardiomyopathy and restrictive atrial septal defect. Laboratory findings revealed a severe, progressive cytolysis syndrome, with negative genetic tests for Duchenne muscular dystrophy and type II glycogen storage disease Pompe disease. Haematological tests excluded a pathology in this section and the serology for viruses with liver tropism was negative.

The particularity of this case lies in the discrepancy between severe cytolysis syndrome and clinical oligosymptomatic presentation.
\end{abstract}

Keywords: hypertrophic cardiomyopathy, cytolysis syndrome, child

\section{INTRODUCTION}

Hypertrophic cardiomyopathy (HCM) is a myocardial disorder defined by an increase in left ventricular wall thickness, with diastolic dysfunction, being most often caused by a mutation in cardiac sarcomere protein genes (cardiac beta-myosin heavy chain gene). In $30-60 \%$ of cases, the disease seems to be genetically transmitted, as an autosomal dominant trait and in the remainder cases, it appears sporadically (1). It is of great value to mention the extreme heterogeneity of the disease: at this point, there are 15 genes encoding contractile sarcomere proteins (myosin, tropomyosin) that can suffer mutations. These genes are located on 16 different chromosomes, reaching over 400 mutations (2). On the other hand, there is chance that infants of diabetic mothers are born with hypertrophic cardiomyopathy, as there is a high prevalence for this group of patients: $10 \%$ to $20 \%$ of them. The prognosis of the disease depends on the etiology that caused the anomaly.
Among paraclinical findings of any HCM, regardless of the cause that have determined it, typical elements of cytolysis syndrome are found.

In pediatric medical practice, increased attention should be paid to the severity of this cytolysis syndrome, because the etiological spectrum is vast and many autoimmune, metabolic and infectious diseases can generate persistently elevated levels of the cytolysis markers, posing serious diagnostic and treatment problems.

According to a recent study conducted by dr. Trandafir L. et al. on a group of 394 children aged 1.5 months to 16 years old, cytolysis syndrome in children is relatively common, having a multifactorial etiology. This study showed that the etiology of cytolysis syndrome is dominated by acute respiratory, digestive and urinary infections, followed by viral infections (Epstein Barr virus and Citomegalovirus). Parasitic infections, obesity or overweight and nutrition and metabolic diseases are also incriminated. In $20.31 \%$ of cases, the etiology was 
not elucidated, the possibilities of serological exploration being limited (3).

\section{CASE PRESENTATION}

We present the case of a two and a half years old male toddler, who was diagnosed with systolic heart murmur and he was admitted to our Pediatric Cardiac Department for establishing the etiology of the heart murmur. The patient's medical history is without clinical significance.

The physical examination of the patient revealed a toddler with hemodynamic stability, with no height or weight delay, no genetic facial features, no motor deficit or retardation, with discrete mucotegumentary pallor, and regarding cardiovascular system: heart rate 98 beats per minute, systolic heart murmur (II/6), blood pressure $90 / 55 \mathrm{mmHg}$, oxygen saturation $98 \%$ on room air. Echocardiographic diagnosis: concentric obstructive hypertrophic cardiomyopathy - with mid-ventricular pressure gradient, mild mitral regurgitation, small restrictive atrial septal defect (Fig. 1 and 2).

Paraclinical findings revealed the elevation of specific liver enzymes alanine transaminase (ALT) and aspartate transaminase (AST): ALT 184-277

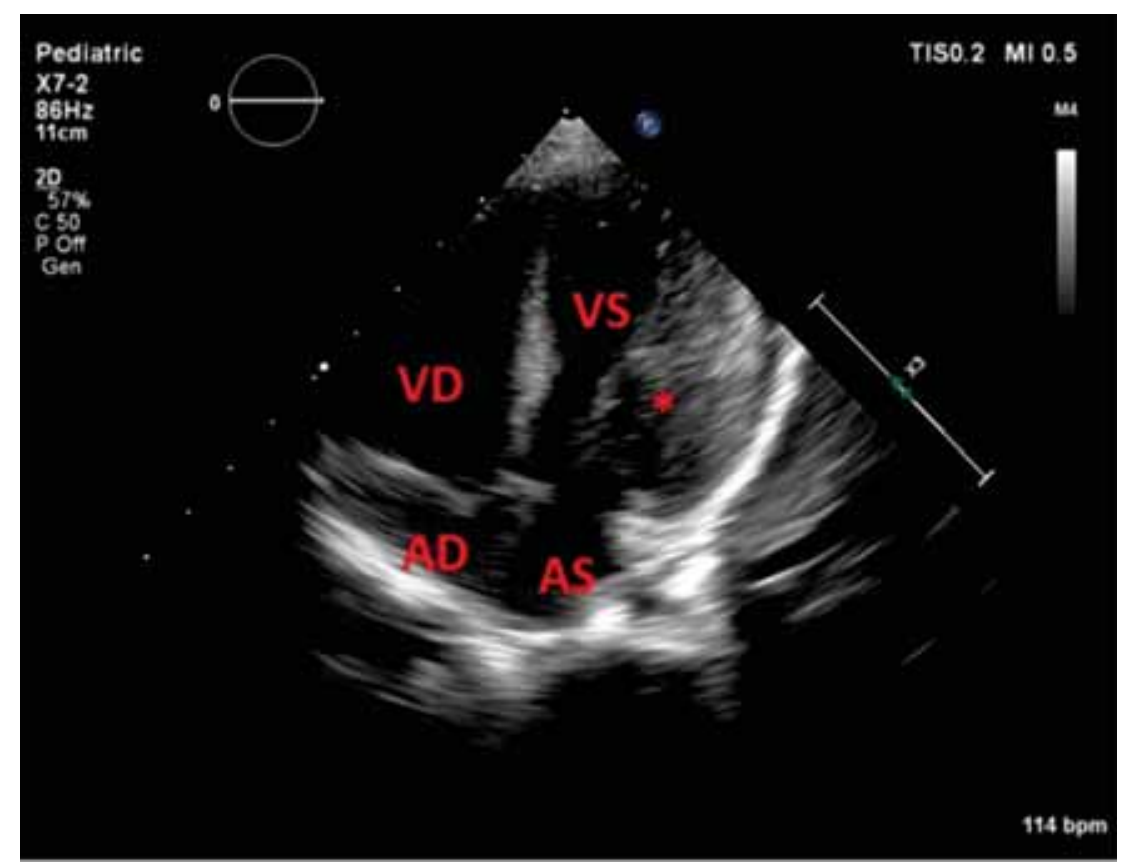

FIGURE 1. Two-dimensional echocardiogram of asymmetric hypertrophy

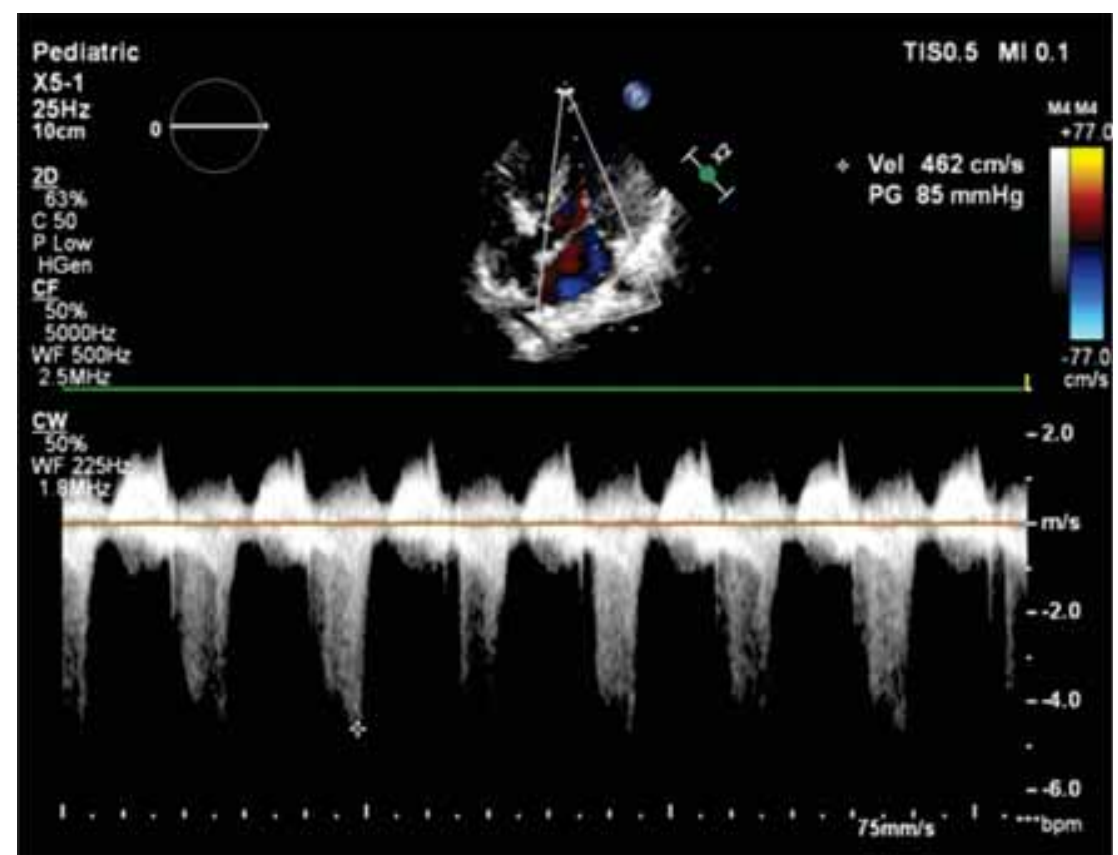

FIGURE 2. Continuous - wave Doppler interrogation from apical 5 chamber showing dynamic left ventricular outflow tract obstruction 
U/1 (up to 5-6 times the normal values) and AST 240-331 U/1 (up to 9-10 times the normal values). Also, we detected high values of serum muscle enzymes: creatine kinase (CK) and lactate dehydrogenase (LDH): CK 390-680 U/1 (up to 4 times the normal values) and LDH 1021-2224 U/1 (up to 5 times the normal values). These high values of serum enzymes are constant during follow-up (during the 2 years since the child is in our evidence), despite the hemodynamic stability, good general condition of the toddler and the treatment set: betablockers (Propranolol) for the control of the mid-ventricular obstruction and hepatoprotective drugs (arginine aspartate - Sargenor, aminoacids Aminosteril-N Hepa, complex of vitamins and aminoacids - Aspatofort and hepatoprotective herbal drugs - Liv 52).

In order to eliminate other causes that may be involved in the etiology of the cytolysis syndrome, the child was admitted to Pediatric Clinic $1 \mathrm{Tg}$. Mures. Here serological determinations for hepatitis $\mathrm{A}, \mathrm{B}$ and $\mathrm{C}$ viruses, Epstein-Barr virus, cytomegalovirus and herpes virus were performed, which were negative. Similarly, hematological disorders were ruled out: thalassemia, hemolytic anemia, also parasitic diseases (including toxoplasmosis). The evaluation for excluding the progressive muscular dystrophy was performed in Pediatric Neurological Clinic „Alexandru Obregia“ in Bucharest, where genetic tests ruled out a dystrophinopathy - Duchenne muscular dystrophy and type II glycogen storage diseases: Pompe disease.

There were several other possible etiologies for the muscular cytolysis syndrome which we have been able to exclude through clinical and paraclinical means: endocrine disorders, neoplasm (acute leukemia), skin disease that can cause falsely increased levels of serum LDH, shock and anoxia, repeated intramuscular injections, use of certain drugs that may cause elevated levels of serum LDH: aspirin, codeine or vitamin A.

The analysis of the acylcarnitine profile was also performed, as it is known that carnitine deficiency can cause hypertrophic / dilated cardiomyopathy.

\section{DISCUSSIONS}

Most studies show that prognosis in hypertrophic cardiomyopathy is influenced by the type of the disease (obstructive or non-obstructive), by the presence of genetic disorders and by recognition of life-threatening cardiac arrhythmias $(4,5,6)$. In the presence of a HCM, a severe cytolysis syndrome with progressive and persistent evolution requires close monitoring in order to eliminate other associated non-cardiac pathologies $(7,8)$. In our case, genetic tests excluded Duchenne muscular dystrophy and type II glycogen storage diseases - Pompe disease.

The difficulty of the case resides in the accuracy of framing the etiology of the cytolysis syndrome and determine what is the weight of the cardiac condition in this equation.

Regarding the cardiac status, the patient had a favorable evolution following treatment with betablockers, without aggravation over time of the echocardiographic parameters or hemodynamic status.

It is already well known that creatine kinase enzyme exists in relatively few organs, being used as a specific index of injury to myocardium and other muscle. According to a study conducted by Swei $\mathrm{H}$. Tsung on a group of 70 patients, although there is evidence of activity of this enzyme in other specific tissues too, the most intense activity is reported in the skeletal muscle and myocardium (9).

Before considering applying invasive investigations (cardiac muscle biopsy and skeletal muscle biopsy) we decided to analyze the five lactate dehydrogenase isoenzymes, as fractionating the $\mathrm{LDH}$ activity sharpens its diagnostic value. The first two isoenzymes are related to cardiac muscle function, the third one is linked to pulmonary injury and the fourth and the fifth ones indicate liver damage (10). The results of the test for our patient revealed the first isoenzyme with values above the normal value (NV): $44.7 \%$ (NV 31\%), specific to heart injury.

In the presence of a severe cytolysis syndrome, there is need to conduct differential diagnosis between cardiac pathology and rhabdomyolysis; the progressive and persistent evolution of this syndrome compels the widening of investigations and remains an unforeseeable element for the prognosis of underlying condition.

The particularity of this case lies in the discrepancy between severe cytolysis syndrome and clinical oligosymptomatic presentation of a child with $\mathrm{HCM}$.

\section{CONCLUSIONS}

Severe and persistent cytolysis syndrome in a child with hypertrophic cardiomyopathy with hemodynamic stability requires careful monitoring, anticipating an unpredictable prognosis. 


\section{REFERENCES}

1. Park Myung K. Primary myocardial diseases (Cardiomyopathy). In: Park Myung K. Park's The pediatric cardiology handbook, 5th ed. Philadelphia: Elsevier Saunders, 2016: 207-219;

2. Ciofu E., Ciofu C. Primitive cardiomyopathies. In: Ciofu E., Ciofu C. Essentials of Pediatrics, 4th ed. Bucharest: Amaltea, 2017: 324-333;

3. Trandafir L., Diaconu G., Straticiuc I. et al. The significance of cytolysis syndrome in children. Ro J Pediatr. 2013; 62(4): 376-381;

4. Maron B.J., Rowin E.J., Casey S.A. et al. Hypertrophic cardiomyopathy in children, adolescents and young adults associated with low cardiovascular mortality with contemporary management strategies. Circulation. 2016; 133(1): 62-73

5. Ziolkowska L., Turska-Kmiec A., Petryka J. et al. Predictors of long-term outcome in children with hypertrophic cardiomyopathy. Pediatr Cardiol. 2016; 37(3): 448-458;
6. Sabater-Molina M., Perez-Sanchez I., Hernandez Del Rincon J.P. et al. Genetics of hypertrophic cardiomyopathy: a review of current state. Clin Genet. 2017;

7. Arad M., Maron B.J., Gorham J.M. et al. Glycogen storage diseases presenting as hypertrophic cardiomyopathy. N Engl J Med. 2005; 352: 362-372.

8. Al-Lozi M.T., Amato A.A., Barohn R.J. et al. Diagnostic criteria for late-onset (childhood and adult) Pompe disease. Muscle nerve. 2009; 40(1): $149-160$

9. Swei H. Tsung. Several conditions causing elevation of serum CK-MB and CK-BB. Am J Clin Pathol. 1981; 75(5): 711-715;

10. Fischbach F. Chemistry studies. In: Fischbach F. A manual of Laboratory Diagnostic Tests, 2nd ed. Maryland: Lippincott Williams \& Wilkins, 1984: 303-310; 